Short Report

\title{
Chimeric Antigen Receptor Therapy for B-cell Malignancies
}

\section{David L Porter ${ }^{1,2 凶}$, Michael Kalos ${ }^{1,3}$, Zhaohui Zheng ${ }^{1,3}$, Bruce Levine ${ }^{1,3}$, Carl June ${ }^{1,3}$}

1. Abramson Cancer Center, University of Pennsylvania Medical Center, Philadelphia, Pennsylvania, 19104, USA

2. Division of Hematology-Oncology, Department of Medicine, University of Pennsylvania Medical Center, Philadelphia, Pennsylvania, 19104, USA

3. Department of Pathology and Laboratory Medicine, University of Pennsylvania Medical Center, Philadelphia, Pennsylvania, 19104, USA

Corresponding author: David L Porter, 3400 Civic Center Boulevard, PCAM 2 West Pavilion, Philadelphia, PA 19104, Phone 2156622862 Fax 2156155888 Email david.porter@uphs.upenn.edu

(C) Ivyspring International Publisher. This is an open-access article distributed under the terms of the Creative Commons License (http://creativecommons.org/ licenses/by-nc-nd/3.0/). Reproduction is permitted for personal, noncommercial use, provided that the article is in whole, unmodified, and properly cited.

Received: 2011.04.21; Accepted: 2011.05.27; Published: 2011.06.01

\begin{abstract}
We presented data showing that the CART-19 cells expressing the 4-1BB signaling domain can have unprecedented and massive in-vivo expansion, traffic to tumor sites, persist long term in vivo, and induce rapid and potent anti-tumor activity in chemotherapy refractory CLL patients.
\end{abstract}

Key words: B-cell Malignancies, CART-19, Chimeric Antigen Receptor Therapy

Targeted tumor-specific cellular therapy will overcome many of the current limitations of adoptive immunotherapy. Gene transfer techniques have now been developed to genetically modify $\mathrm{T}$ cells to confer novel antigen specificity by stably expressing a chimeric antigen receptor (CAR) on their surface. CARs combine an antigen recognition domain of a specific antibody with an intracellular domain of the CD3-zeta chain or FcyRI protein into a single chimeric protein ${ }^{1}$. When antigen is encountered, CAR-modified T cells become activated and kill in an antigen dependent, but HLA independent manner, making this an attractive approach as a generalized cancer therapy ${ }^{2}$. Until recently, development of this technology has been limited by lack of efficient $T$ cell culture systems and gene transfer techniques. Therefore, most clinical applications have resulted in limited in vivo expansion and persistence of CAR-modified $\mathrm{T}$ cells resulting in disappointing clinical activity ${ }^{3,4}$.

CD19 is an ideal tumor target. Expression is restricted to B cells, from the pro-B cell stage to mature
B cells (though not on plasma cells), possibly follicular dendritic cells, and it is expressed on the surface of most B cell malignancies. Importantly, it is not expressed on pluripotent marrow stem cells 5 .

We have combined a robust $T$ cell culture system $^{6}$ with lentiviral vector transduction of human $\mathrm{T}$ cells to express a CD19-specific CAR (CART-19 cells). The signaling domain of the CAR is critical for activation of cytolytic activity and survival. First-generation CARs typically utilized the intracellular domain of the CD3z chain only, the primary signaling molecule from endogenous T-cell receptors. Second generation CARs had a more potent signaling domain from various co-stimulatory receptors such as CD28 or CD137 (4-1BB). Preclinical studies showed that inclusion of potent signaling molecules improves the antitumor activity of genetically modified T cells. Our group has tested a CAR directed against CD19 linked to the CD137 (4-1BB) co-stimulatory molecule signaling domain to enhance activation and signaling after recognition of CD19. By inclusion of the 4-1BB sig- 
naling domain, in vitro tumor cell killing, and in-vivo anti-tumor activity and persistence of CART-19 cells in a murine xenograft model of human ALL is greatly enhanced 7 .

Given these preliminary findings, we have initiated a clinical trial to test the feasibility and safety of CART-19 cells in patients with CD19+ lymphoid malignancies. Our initial patients all had advanced, refractory CLL (2 with deletion of chromosome 17p) and were considered incurable with standard therapies. All patients receive lymphodepleting chemotherapy 1-4 days prior to CART-19 infusions. Lymph node depletion has been necessary for most models of adoptive immune therapy. This may permit homeostatic proliferation of the infused $\mathrm{T}$ cells, eliminate competitive $\mathrm{T}$ cells that could serve as a "sink" for important activating cytokines, result in elimination of regulatory and suppressive $\mathrm{T}$ cells or even enhance endogenous host APC activity ${ }^{8}$.

For manufacturing, CD3+CD45+ cells in a leukapheresis product are positively selected with anti-CD3/anti-CD28 magnetic beads prior to CART-19 lentiviral vector transduction. After transduction, cells are expanded ex-vivo and patients are infused with a maximum targeted dose of $5 \times 10^{9}$ total T cells; approximately $10-30 \%$ of cells will express the anti-CD19 CAR.

Our preliminary findings were presented at the $4^{\text {th }}$ Autologous and Allogeneic Cell Therapy in Solid Tumors meeting and show that in the initial few patients, manufacturing was feasible and infusions were well tolerated. We presented data showing that the CART-19 cells expressing the 4-1BB signaling domain can have unprecedented and massive in-vivo expansion, traffic to tumor sites, persist long term in vivo, and induce rapid and potent anti-tumor activity in chemotherapy refractory CLL patients. We have not observed clonal proliferation of the infused $\mathrm{T}$ cells with over 6 months follow-up of treated patients. We, and others 4 , have observed eradication of B cells and hypogammaglobulinemia in some patients raising concern for long-term immune deficiency, particularly if the CART-19 cells persist for long periods of time. However, to date, no toxicity from B cell deficiency has been noted and repletion of intravenous gammaglobulin can be used to minimize risks associated with hypogammaglobulinemia. The development of a cytokine release syndrome in first patients treated highlights the need for caution and continued study of this promising new therapy. Treatment of additional patients with detailed analyses and longer follow up will be needed to define the ultimate role of this therapy.
The potent activity demonstrated in our first few patients is extremely encouraging and suggests that autologous $\mathrm{T}$ cells genetically modified with redirected specificity for $\mathrm{CD} 19$, with the appropriate signaling domain, may offer significant advantages over existing therapies, including allogeneic SCT. Although the clinical application of CAR-modified T cells is in its infancy ${ }^{9}$, there has been remarkable progress in the last few years, and we believe this therapy holds great promise as a potent and sustained method of cancer immunotherapy.

\section{Acknowledgement}

This work was supported in part by grants from The Leukemia \& Lymphoma Society (7000-02, DLP, $\mathrm{CHJ}$ ) and NIH (K24 CA11787901) (DLP).

\section{Conflict of Interest}

The authors have declared that no conflict of interest exists.

\section{References}

1. Irving BA, Weiss A. The cytoplasmic domain of the T cell receptor zeta chain is sufficient to couple to receptor-associated signal transduction pathways. Cell. 1991;64:891-901.

2. Sadelain M, Brentjens R, Riviere I. The promise and potential pitfalls of chimeric antigen receptors. CurrOpinImmunol. 2009;21:215-223.

3. Lamers $\mathrm{CH}$, Willemsen $\mathrm{R}$, van Elzakker $\mathrm{P}$, et al. Immune responses to transgene and retroviral vector in patients treated with ex vivo-engineered T cells. Blood 2011;117:72-82.

4. Kochenderfer J, Wilson W, Janik J, et al. Eradication of B-lineage cells and regression of lymphoma in a patient treated with autologous $\mathrm{T}$ cells genetically-engineered to recognize CD19. Blood. 2010;116(20):4099-102.

5. Uckun FM, Jaszcz W, Ambrus JL, et al. Detailed studies on expression and function of CD19 surface determinant by using B43 monoclonal antibody and the clinical potential of anti-CD19 immunotoxins. Blood. 1988;71:13-29.

6. Levine BL, Bernstein WB, Connors $\mathrm{M}$, et al. Effects of CD28 costimulation on long-term proliferation of CD4+ T cells in the absence of exogenous feeder cells. Journal of Immunology. 1997;159:5921-5930.

7. Milone MC, Fish JD, Carpenito C, et al. Chimeric receptors containing CD137 signal transduction domains mediate enhanced survival of $\mathrm{T}$ cells and increased antileukemic efficacy in vivo. Molecular Therapy: the Journal of the American Society of Gene Therapy. 2009;17:1453-1464.

8. Cui $Y$, Zhang H, Meadors J, et al. Harnessing the physiology of lymphopenia to support adoptive immunotherapy in lymphoreplete hosts. Blood. 2009;114:3831-3840.

9. Kohn D, Dotti G, Brentjens R, et al. CARs on Track in the Clinic: Workshop of the Blood and marrow Transplant Clinical Trials Network Subcommittee and Cell and Gene Therapy. Molecular Therapy: the Journal of the American Society of Gene Therapy. 2011;19:432-438. 